\section{Einladung zur Tagung der International Cancer Imaging Society (ICIS) im Herbst in Berlin}

Sehr geehrte Kolleginnen und Kollegen,

nach 2014 (Heidelberg, Kongresspräsident Prof. Schlemmer) wird die Tagung der „International Cancer Imaging Society (ICIS)“ in 2017 zum zweiten Mal in Deutschland stattfinden, nämlich vom 02.-04.Oktober 2017 (d. h. über den Feiertag am 3. Oktober).

Die Tagung beschäftigt sich ausschließlich mit Bildgebung und radiologischen Interventionen bei onkologischen Patienten.

Neben Vorträgen zu innovativen Verfahren, Refresherkursen zum state-of-the-art werden verschiedene interaktive Formate beim Kongress angeboten: Interaktive Hands-on Workshops mit max. 42 Teilnehmern an Befundungsworkstations zu acht verschiedenen Themen der onkologischen Bildgebung (Lunge, Mamma, Prostata, Leber, Pankreas, Ovar, Uterus und Ganzkörper-MRT) und live-case Workshops in Zusammenarbeit mit der Charité und der Evangelischen Lungenklinik Berlin zu onkologischen Interventionen (Mikrowellenablation, Radiofrequenzablation, TACE, SIRT).

Hierbei werden Teilnehmer in Kleingruppen in den Kliniken die Fälle diskutieren und bei der Therapie im Raum sein. Dieses Format ist besonders für die Kollegen empfehlenswert, die ihre Erfahrungen in den jeweiligen Interventionen vertiefen oder die Methoden in ihren Einrichtungen neu etablieren wollen.
Es wird wissenschaftliche Sitzungen und eine Posterausstellung geben; die Abstract-Einreichung ist ab sofort geöffnet (Deadline 29. Mai 2017). Für den besten wissenschaftlichen Vortrag und das beste Poster werden Preise verliehen werden.

Die Deutsche Röntgengesellschaft hat für DRG-Mitglieder in der Weiterbildung zehn Kongress-Stipendien à 500, - ausgelobt, die einen wissenschaftlichen Vortrag oder ein Poster bei der Tagung der ICIS vorstellen.

Die Bewerbung um ein solches Stipendium soll bei der Abstract-Einreichung auf der Registrierungs-Website der ICIS mitgeteilt werden. Die Entscheidung über die Annahme des Abstracts und (bei mehr als $10 \mathrm{Be}$ werbern) die Gewährung eines Stipendiums trifft das wissenschaftliche Komitee der ICIS in Abstimmung mit der DRG.

Um den Kongressbesuch in Berlin noch lohnender zu gestalten, findet am Samstag (30.09.2017) vor der Tagung der ICIS (2.4.10.2017) eine eintägige Fortbildungsveranstaltung der AG Onkologische Bildgebung zu den Themen Bildgebung von Prostata, Mamma und malignem Lymphom statt.

Die drei Themenblöcke sind jeweils gleichartig aufgebaut (Bildgebung, Therapie, interdisziplinäre Fallbesprechung).

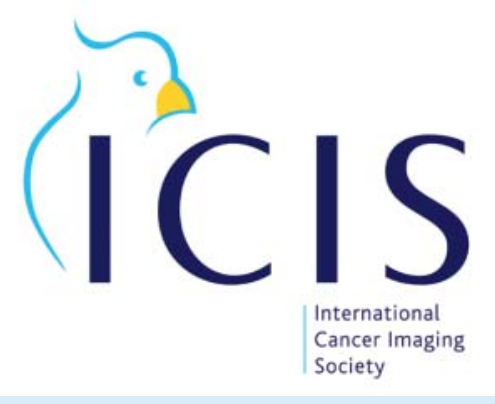

Teilnehmer des ICIS Kongresses können diese Veranstaltung zu einer auf $50 \%$ reduzierten Teilnehmergebühren besuchen.

Wir würden uns sehr freuen, wenn das Programm beider Veranstaltungen Ihr Interesse fände.

Mit freundlichen kollegialen Grüßen

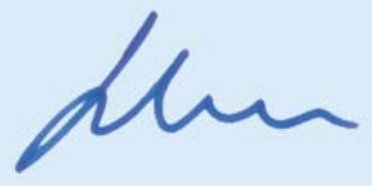

Prof. Dr. med. Heinz-Peter Schlemmer, Vorsitzender der AG Onkologische Bildgebung der DRG

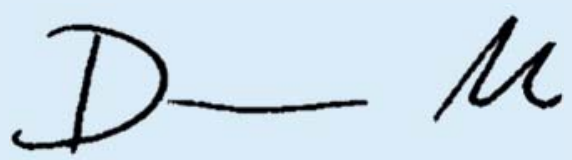

Prof. Dr. med. Stefan Diederich, ICIS Präsident 